\title{
Évaluer les mécanismes de défense aujourd'hui
}

\author{
Evaluating defensive mechanisms today
}

\author{
A. Braconnier \\ (C) Springer-Verlag France 2011
}

Afin d'expliciter au mieux sa compréhension des « psychonévroses de défense », Freud avait progressivement retenu une dizaine de mécanismes de défense [9]. Pour le fondateur de la psychanalyse, la fonction de ces mécanismes était de protéger le Moi contre les exigences pulsionnelles internes et inconscientes : «procédés psychiques dont se sert le Moi dans les conflits susceptibles d'aboutir à une névrose » (1896). Anna Freud, dans son célèbre ouvrage « le Moi et les mécanismes de défense » [8], élargit cette fonction à l'adaptation du Moi à la réalité extérieure. Ce saut est important, car il marque une des évolutions contemporaines majeures qui amènent à rapprocher le terme de mécanisme de défense de celui de coping (faire face), issu du champ cognitif. Mélanie Klein et W. Bion s'intéressèrent, quant à eux, aux défenses précoces ou de « derniers recours ». Plus récemment, Jean Bergeret, Jacques Lacan ou Otto Kernberg mirent en exergue les défenses psychopathologiques chez les patients limites et psychotiques.

Plusieurs débats sur les stratégies défensives sont toujours d'actualité dans le champ de la psychanalyse, au-delà de celui concernant la question ancienne du statut du refoulement :

- quelle place doit-on donner aux mécanismes de défense par rapport aux résistances au traitement et à la dynamique transférentielle/contre-transférentielle ?

- La clarification des stratégies défensives «privilégiées » pour un patient donné doit-elle être faite et constituer un temps initial du traitement permettant une évaluation psychopathologique suffisamment fine et une indication sur les difficultés à prévoir dans le processus thérapeutique ? Ou doit-on d'abord et avant tout s'intéresser aux fantasmes inconscients?

- L'intérêt porté au Moi doit-il être toujours équilibré par rapport à celui porté au Ça et au Surmoi ?

A. Braconnier $(\bowtie)$

Centre Philippe-Paumelle, Association Insight,

11, rue Albert-Bayet, F-75013 Paris, France

e-mail : Alain.braconnier@asm13.org
- Comment ce concept suscite des questions sur l'articulation « réalité interne-réalité externe »?

Toutefois, le fait le plus marquant est l'intégration récente du concept de "mécanisme de défense » dans le champ scientifique de la psychologie, des théories du développement, de la recherche psychopathologique et des classifications psychiatriques internationales. Il faut ici citer deux pionniers dans les études scientifiques contemporaines sur ce sujet : Michael Bond et Georges Vaillant à l'origine de deux importants articles publiés par le premier en 1983 et par les deux en 1986 dans la revue Archives of general of psychiatry qui n'est clairement pas une revue de psychanalyse : « Il est temps que le Moi et ses défenses soient vus comme des facettes de la réalité psychologique, biologique et sociale et non pas comme des objets du culte psychanalytique » écrira Georges Vaillant dix ans plus tard. Ces deux cliniciens et chercheurs sont à l'origine du développement de l'élaboration d'instruments d'évaluation clinique plus facilement utilisables à ce niveau que les tests projectifs et, par là même, de la possibilité des recherches empiriques sur ce sujet.

Un autre fait marquant contemporain est l'introduction d'une annexe sur ce sujet au sein du DSM, sous la forme d'un glossaire dans le DSM-III-R, et d'une échelle de fonctionnement défensif dans le DSM-IV où les mécanismes de défense sont assimilés aux mécanismes d'ajustement ou de coping. Le futur DSM-V semble vouloir garder la même ligne à ce sujet.

L'étude empirique des mécanismes de défense ne concerne donc plus seulement la recherche psychanalytique au sens strict, mais vise à améliorer les connaissances sur le fonctionnement mental intrapsychique, cognitif et peut-être biologique de chacun. Ces études empiriques ont des implications cliniques, thérapeutiques et bien évidemment de recherches.

\section{Instruments contemporains d'évaluation}

Citons les instruments d'évaluation les plus fréquemment utilisés. 


\section{Autoquestionnaires}

- Le Defense Mechanism Inventory (DMI) [10] s'appuie sur 12 histoires qui permettent de dégager cinq styles défensifs. Il n'y a pas de traduction française ;

- le Defense Style Questionnaire (DSQ) [1]. Il existe une forme à 88 items traduite en français [4], regroupant 24 mécanismes de défense en quatre styles correspondant aux quatre facteurs isolés par une analyse factorielle et une forme à 40 items traduite en français par J.-D. Guelfi (2000) confirmant l'analyse factorielle regroupant trois facteurs : style mature, névrotique, immature. Cet autoquestionnaire est généralement considéré comme le plus pratique à utiliser en clinique ;

- pour les adolescents, signalons la Response Evaluation Measure (REM-71) [14] ;

- citons aussi le Defense Mechanism Profile et le Life Index Style.

Évidemment, ici, une question reste : une auto-observation psychique de soi est-elle fiable?

\section{Échelles d'évaluation}

- Le pionnier dans ce domaine, Georges Vaillant, a proposé la Vaillant's Life Vignette. Cet outil identifie les trois défenses prédominantes et évalue la maturité défensive ;

- la Defense Mechanism Rating Scales (DMRS) [13] : elle est la plus représentative dans les travaux actuels de recherche. Elle représente également la grille de référence pour l'annexe B du DSM-IV qui identifie sept niveaux dominants de fonctionnement défensif. La DMRS qui s'appuie sur des enregistrements audio ou vidéo des entretiens ou des séances de psychothérapie. Elle nécessite la formation des cotateurs. Elle permet une évaluation qualitative déterminant la présence ou l'absence de chaque mécanisme de défense et une évaluation quantitative indiquant la fréquence de la mise en oeuvre de chacun des mécanismes de défense. En fin de compte, cette échelle détermine un " profil défensif » et un « score défensif global » pour le sujet.

En comparant ces différentes méthodes d'évaluation, on peut dire que les autoquestionnaires ont l'avantage de la simplicité d'utilisation mais reposent sur une évaluation subjective et consciente du sujet. Le DSQ correspond à une échelle unidimensionnelle mesurant un niveau défensif global. Il existe une nouvelle version du DSQ plus compatible avec le DMRS. Il comprend 60 items et mesure 30 défenses (Y. de Roten, G. Ambresin, 2009). Les outils d'évaluation, quant à eux, sont plus complexes mais prennent mieux en compte l'implicite. Ainsi, le DMRS se montre plus valide et plus sensible sur le plan empirique. Il se montre également plus sensible au changement dans l'évaluation des effets des traitements.

Si l'on se tourne maintenant sur la question de l'utilisation de l'évaluation des mécanismes de défense dans la recherche contemporaine, on s'aperçoit qu'elle a facilité plusieurs types d'études cliniques et thérapeutiques.

\section{En recherche clinique}

Nous citerons les études suivantes :

- étude portant sur le développement chez l'enfant de l'apparition et de la présence des différents mécanismes de défense, en particulier le déni, la projection et l'identification [6] ;

- étude de corrélations avec différentes situations de la vie (deuil, séparation, état amoureux, travail, maladie somatique, etc.) ;

- possibilité de recherches programmées sur les corrélations entre les mécanismes de défense et les diagnostics catégoriels, en particulier épisode dépressif majeur et troubles anxieux. À titre d'exemple, citons la recherche sur la stabilité ou non des mécanismes de défense dans l'histoire des sujets présentant à un moment un état dépressif. La question, ici, était de savoir si les mécanismes de défense constituaient un trait stable de la personnalité ou étaient « état dépendant ». Bond et Vaillant [2] ont constaté alors que l'amélioration du fonctionnement défensif est prédictive de l'amélioration de la dépression. Citons aussi l'étude montrant des corrélations des scores DSQ regroupés en trois facteurs (« mature », « névrotique », « immature »), avec l'évaluation de l'anxiété et de la dépression sur le SCL-90 (hommes, femmes, groupe total) ;

- recherche sur les corrélations entre les résultats de la DMRS et les différentes populations psychiatriques : paranoïaque $(100 \%$ des sujets présentent une « projection », $75 \%$ « le passage à l'acte »), narcissique $(83 \%$ présentent un «déni », $61 \%$ le mécanisme « passage à l'acte » et $39 \%$ la « projection »);

- une étude française a porté sur la prévention du risque suicidaire en rapport avec la DMRS (E. Corruble et al.) ;

- la possibilité de recherches programmées sur les corrélations avec les personnalités, en particulier borderline [3], a été envisagée à plusieurs reprises, mais aussi avec d'autres modèles de personnalité par exemple de type cloninger [10].

\section{En recherche thérapeuthique}

L'évaluation des mécanismes de défense représente un facteur clé dans l'évaluation des indications et du déroulement 
des psychothérapies analytiques et même des psychanalyses proprement dites [5]. Citons ici l'importance des travaux de l'équipe de Lausanne utilisant la DMRS [7]. Cette équipe s'intéresse tout particulièrement à l'évaluation du déroulement favorable du processus psychothérapique. Ils ont par exemple étudié la corrélation avec l'alliance thérapeutique comparant les interventions « intellectualisantes » (sublimation, intellectualisation) aux interventions « confrontantes » (projection, dépréciation ou défenses de dernier recours). Cette équipe évaluant la stabilité et les changements dans les "Interventions psychodynamiques brèves » a par exemple constaté une augmentation des mécanismes de type « obsessionnel » et une diminution des mécanismes de défense dits narcissiques grâce à ce type de traitement.

\section{Dans le champ psychosocial}

Nous savons aujourd'hui qu'il existe un rapport entre les "styles défensifs » évalués par la DMRS et le fonctionnement social évalué par l'EGF.

\section{Conclusion}

Le concept de mécanisme de défense demeure un champ ouvert d'exploration en raison de l'hétérogénéité des définitions, des fonctions et du nombre de mécanismes de défense reconnus. En outre, la question se pose de savoir si l'on doit privilégier l'étude de chaque mécanisme pris un à un ou plutôt des « styles défensifs » regroupant plusieurs mécanismes hiérarchisés et mieux corrélés avec la clinique.

Quelle qu'en soit la réponse, on peut constater que l'étude empirique des mécanismes de défense ou de coping (assimilés dans le DSM-IV) est aujourd'hui possible. Les autoquestionnaires sont rapides et faciles à passer en clinique et permettent de mieux connaître le fonctionnement du sujet présentant un trouble psychique ou, par exemple, une maladie somatique. Ils peuvent aussi être utilisés chez les soignants, déterminants ainsi les questions institutionnelles, individuelles ou surtout groupales.

Ces études posent néanmoins plusieurs questions, notamment celle du statut scientifique de la psychanalyse, soulevant les difficultés posées par l'évaluation de processus psychiques, par nature inconscients. Ici, de quels « inconscients » parle-t-on ? D'inconscient cognitif (préconscient pour les psychanalystes) ou d'inconscient psychanalytique proprement dit? Une autre question est bien entendu celle des interrelations entre la psychiatrie et la psychanalyse (notons à ce propos les opinions contrastées selon que l'on est dans le champ de la psychanalyse anglo-saxonne ou française : empirisme versus théorie.).

Les études sur ces mécanismes dépassent largement le cadre de la pathologie psychiatrique proprement dite. Elles correspondent toujours à un intérêt en clinique psychopathologique et peuvent aussi devenir des outils de recherche dans l'évaluation de l'efficacité des différents types de psychothérapies mais aussi de traitements médicamenteux. Elles peuvent également éclairer les retentissements des stratégies défensives de chacun sur l'évolution des maladies somatiques.

\section{Références}

1. Bond MP, Gardner ST, Christian J, et al (1983) Empirical study of self rated defense styles. Arch Gen Psychiatry 40:333-8

2. Bond MP, Vaillant GE (1986) An empirical study of relationship between diagnosis and defense style. Arch Journ Psychiatry 43:285-88

3. Bond MP (1994) Defense style and borderline personality disorder. J Personal Disord 8:28-31

4. Bonsack CH, Despland JN, Spagnoli J (1998) The French version of the Defense Style Questionnaire. Psychother Psychosom 67:24-30

5. Brenner C (1975) Alterations in defenses during psychoanalysis. In: Waldhorn HF, Fine BD (eds) International University Press, New York

6. Cramer P (1991) The development of defense mechanisms: theory, research and assessment. Springer-Verlag, New York

7. Desplands JN, Michel L, de Roten Y, et al (2010) L'intervention psychodynamique brève. Masson-Elsevier, Paris

8. Freud A (1936) Le Moi et les mécanismes de défense. Presses Universitaires de France, Paris

9. Freud S (1896) «Les psychonévroses de défense » et « Nouvelles remarques sur les psychonévroses de défense » dans Névrose, psychose et perversion. Presses Universitaires de France, Paris

10. Gleser GC, Ihlevich D (1969) An objective instrument for measuring defense mechanisms. J Consult Clin Psychol 33:51-60

11. Mulder RT, Joyce PR, Sellman JD, et al (1996) Towards an understanding of defense style in terms of temperament and character. Acta Psychiatrica Scandinavia 93: 99-104

12. Perry JC (1990) Psychological defense mechanisms and the study of affective and anxiety disorders. In: Maser JD, Cloninger CR (eds) Comorbidity of mood and anxiety disorder. Karger, Washington, DC

13. Perry JC (2009) Échelles d'évaluation des mécanismes de défense (deuxième édition). Traduit et commenté par JD Guelfi, JN Despland, B Hanin, Masson, Paris

14. Steiner MD, Araujo KB, Koopman C (2001) The Response Evaluation Measure (REM-71). A new instrument for the measurement of defenses in adults and adolescents. Am J Psychiatry 158:467-73

15. Sultanian C, Dardennes R, Mouchabac S, et al (2005) L'évaluation normalisée et clinique des mécanismes de défense ; revue critique de 6 outils quantitatifs. Rev Can Psychiatrie 50:792-801

16. Vaillant GE, Bond M, Vaillant CO (1986) An empirical validated hierarchy of defense mechanisms. Arch Gen Psychiatry 43:786-94 